\title{
ATLAS Trigger and DAQ Upgrades for High-Luminosity LHC
}

\author{
M. ISHINO on behalf of the ATLAS collaboration ${ }^{1, a}$ \\ ${ }^{1}$ ICEPP, The University of Tokyo, Bunkyo-ku Hongo 7-3-1, Tokyo, Japan
}

\begin{abstract}
The ATLAS Trigger-DAQ system will be significantly upgraded to explore new physics beyond the standard model in the High-Luminosity LHC environment with instantaneous luminosity of up to $7.5 \times 10^{34} \mathrm{~cm}^{-2} \mathrm{~s}^{-1}$. Two general trigger schemes are under study and their prospects and concerns are discussed. The muon trigger and readout electronics will be totally replaced to cope with a longer latency and a higher trigger rate. At the same time, new trigger algorithms using the precision tracker will be introduced to sharpen efficiency turn-ons and suppress fake triggers. The algorithm and the expected performance of the muon trigger are described. The hardware based track trigger is one of the major items introduced in the upgrade program. It will improve the trigger performance of various trigger items with a combination of the calorimeter or muon signature. The procedure for processing track information and an expected performance on the electron trigger are presented.
\end{abstract}

\section{Introduction}

The ATLAS Collaboration [1] is planning make significant upgrades to the trigger-DAQ system to achieve the best reach to new physics beyond the standard model in the High-Luminosity LHC program. The system is designed to maintain the trigger thresholds at the peak luminosity of $7.5 \times 10^{34} \mathrm{~cm}^{-2} \mathrm{~s}^{-1}$ at similar levels to the LHC Run-1 period when the instantaneous luminosity was $0.7 \times 10^{34} \mathrm{~cm}^{-2} \mathrm{~s}^{-1}$ and keep the physics acceptance as large as possible.

A simplified example of the trigger menu is shown in Table 1. The list of trigger items with corresponding thresholds for offline analyses, geometrical acceptance in pseudo-rapidity as well as trigger rates for each trigger level at a luminosity of $7.5 \times 10^{34} \mathrm{~cm}^{-2} \mathrm{~s}^{-1}$ and $\sqrt{\mathrm{s}}=14 \mathrm{TeV}$ are presented. As an example the single lepton "isolated single electron" and "single $\mu$ " objects have $p_{T}$ thresholds of $22 \mathrm{GeV}$ and $20 \mathrm{GeV}$ respectively, compared to the Run-1 threshold of $25 \mathrm{GeV}$.

Two options, a single-level and a two-level scheme, are under consideration, described in Section 2. The latency of the hardware trigger and the maximum output rate of the hardware and software triggers will be increased, with latencies of $10 \mu$ s for the Level- 0 trigger and $60 \mu \mathrm{s}$ for the Level-1 trigger allowing the hardware trigger to use similar algorithms to the current high-level trigger.

Information from the precision Monitored Drift-Tube (MDT) tracker will be used in the Level-0 muon trigger. The sharp turn-on of efficiency in terms of muon $p_{T}$ of the MDT trigger will allow effective rejection of candidates below threshold, controlling the rate, whilst maximising the trigger efficiency. The general scheme of the Level-0 muon trigger is discussed in Section 3.

ae-mail: Masaya.Ishino@cern.ch 
Table 1. Simplified and illustrative trigger menu at an instantaneous luminosity of $7.5 \times 10^{34} \mathrm{~cm}^{-2} \mathrm{~s}^{-1}$. There are considerable overlaps in the rates, especially for the hadronic triggers. The totals also allow for pre-scaled supporting triggers which are not listed for the tables. For hadronic items, the Event Filter rates assume additional rejection from b-tagging and other event-level analysis algorithms. Forward jets also include multi jet triggers with lower thresholds and requirements on other quantities such as invariant mass.

\begin{tabular}{|l|c|c|r|r|r|}
\hline Item & $\begin{array}{c}\text { Offline } p_{T} \\
\text { Threshold } \\
{[\mathrm{GeV}]}\end{array}$ & Offline $|\eta|$ & $\begin{array}{r}\text { L0 } \\
\text { Rate } \\
{[\mathrm{kHz}]}\end{array}$ & $\begin{array}{r}\text { L1 } \\
\text { Rate } \\
{[\mathrm{kHz}]}\end{array}$ & $\begin{array}{r}\mathrm{EF} \\
\text { Rate } \\
{[\mathrm{kHz}]}\end{array}$ \\
\hline isolated single $e$ & 22 & $<2.5$ & 200 & 40 & 2.20 \\
forward $e$ & 35 & $2.4-4.0$ & 40 & 8 & 0.23 \\
single $\gamma$ & 120 & $<2.4$ & 66 & 33 & 0.27 \\
single $\mu$ & 20 & $<2.4$ & 40 & 40 & 2.20 \\
di- $\gamma$ & 25 & $<2.4$ & 8 & 4 & 0.18 \\
di-e & 15 & $<2.5$ & 90 & 10 & 0.08 \\
di- $\mu$ & 11 & $<2.4$ & 20 & 20 & 0.25 \\
$e-\mu$ & 15 & $<2.4$ & 65 & 10 & 0.08 \\
single $\tau$ & 150 & $<2.5$ & 20 & 10 & 0.13 \\
di- $\tau$ & 40,30 & $<2.5$ & 200 & 30 & 0.08 \\
single jet & 180 & $<3.2$ & 60 & 30 & $0.60^{*}$ \\
large- $R$ jet & 375 & $<3.2$ & 35 & 20 & $0.35^{*}$ \\
four-jet & 75 & $<3.2$ & 50 & 25 & $0.50^{*}$ \\
$H_{T}$ & 500 & $<3.2$ & 60 & 30 & $0.60^{*}$ \\
$E_{T}^{\text {miss }}$ & 200 & $<4.9$ & 50 & 25 & $0.50^{*}$ \\
jet $+E_{T}^{\text {miss }}$ & 140,125 & $<4.9$ & 60 & 30 & $0.30^{*}$ \\
forward jet ${ }^{* *}$ & 180 & $3.2-4.9$ & 30 & 15 & $0.30^{*}$ \\
\hline Total & & & $\sim 1000$ & $\sim 400$ & $\sim 10$ \\
\hline
\end{tabular}

In the Level-1 trigger, track information from the ATLAS inner tracker will be available on the hardware trigger. Combining this with the output of the Level-0 calorimeter and muon signature will significantly reduce the trigger rate of a number of trigger items. As an example the expected performance of the single electron trigger is described in Section 4, and a summary is given in the last section.

\section{The general schemes of the ATLAS Trigger and DAQ}

A new trigger and DAQ scheme will be introduced for the high-luminosity LHC program (HL-LHC) to maintain trigger thresholds similar to those in the Run-1 period. Two options on the trigger and DAQ scheme are under study and they are presented in Figure 1. The first option in Figure 1-(a) consists of two hardware trigger levels and the Event Filter (EF) with the associated data readout path (DAQ). The second option has a single hardware trigger level and all the accepted events are sent to the EF and DAQ as shown in Figure 1-(b).

\subsection{Two-level hardware trigger scheme}

In the two-level hardware scheme, a Level-0 trigger rate of $1 \mathrm{MHz}$ and a Level-1 trigger rate of $400 \mathrm{kHz}$ are assumed. The Level-0 trigger latency is set to $10 \mu \mathrm{s}$ and the Level-1 trigger latency is set to $60 \mu \mathrm{s}$. Following the diagram in the Figure 1-(a) from top to bottom, the calorimeter and muon detector information is provided to the Level-0 calorimeter (LOCalo) and muon trigger (LOMuon). 


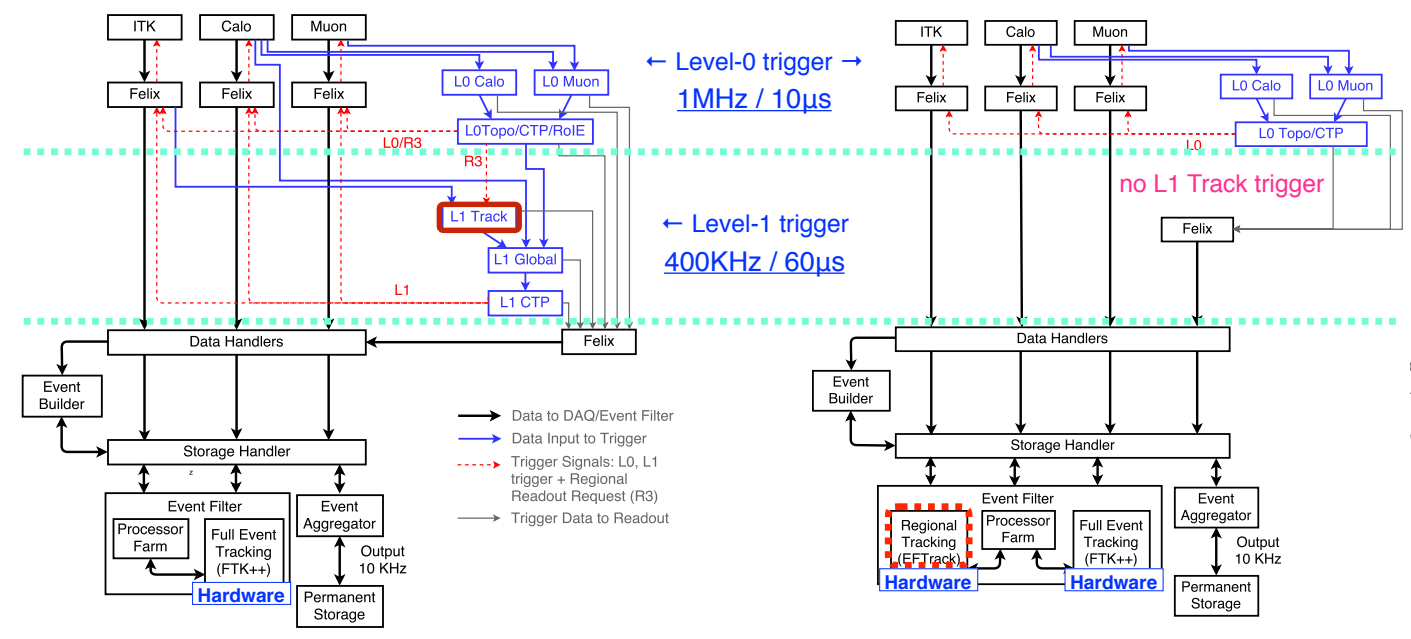

(a)

(b)

Figure 1. High-level design diagram of the ATLAS trigger and DAQ system is shown. The two hardware level scheme in the Figure (a), while single level hardware scheme which does not include Level-1 trigger is shown in Figure (b). The maximum trigger rate and the maximum possible latency are indicated.

The LOCalo trigger identifies and measures the energy of electron, tau, jet candidates and calculates missing transverse-energy $\left(E_{T}^{m i s s}\right)$. The LOMuon trigger finds muon tracks in the spectrometer and processes and determines their transverse-momentum $\left(p_{T}\right)$. A new feature of the LOMuon trigger for HL-LHC is to include the precision tracker, MDT, information to determine the $p_{T}$ of muon candidates. The Level-0 Topological trigger (L0Topo) receives a list of objects from L0Calo and L0Muon together with the information of energy and location (Region-of-Interest : RoI) in the detector, and then combines them to more complex signatures, for example invariant mass, opening angle and so on. The Level-0 Central Trigger Processor (L0CTP) receives the output of L0Topo and makes a final Level-0 decision. A pre-scale factor assigned to each trigger item and dead-time handling are taken care by the LOCTP system.

The Region-of-Interest Engine (RoIE) processes the R3 (Regional-Readout-Request) signal according to the information given by LOCTP and L0Topo and provides R3 to the data readout system (FELIX). A part of the inner-tracker (ITK) data and the calorimeter information specified in the R3 signal are sent to the Level-1 track trigger (L1Track) and the Level-1 global trigger (L1Global). The L1Track trigger finds tracks and estimates momenta on the special hardware and the output is passed to the L1Global trigger. The L1Global trigger combines the track information and the finer- 
grained calorimeter information, and then processes refined signatures of electrons, photons, taus, jets, $E_{T}^{\text {miss }}$ and other hadronic signatures. The Level-1 Central Trigger Processor (L1CTP) receives the output of L1Global and makes a final Level-1 decision according to the trigger menu configuration and pre-scales.

Depending on the detector system the full event data are readout by FELIX on either the Level-0 or Level-1 trigger accept signal and transmitted to the Data Handler, where raw data is formatted. The Storage Handler receives the full set of event data and buffers until the Event Filter (EF) makes a final decision to record the event or not.

The Event Filter (EF) is placed in the last step of the trigger chain and selects events to be finally recorded or not. The EF system consists of a large scale computing farm and a dedicated hardware tracking system $(\mathrm{FTK}++)$ which provides a full track list using hit information of the ITK detectors. Because of the high track density in a single event, the help of special hardware is required. The event accept rate of the $\mathrm{EF}$ is $10 \mathrm{kHz}$.

The event data selected by the EF system is transferred from the Storage Handler to the Permanent Storage system by the Event Aggregator. The size of the raw data is estimated as $5 \mathrm{MB}$ per event. The DAQ system needs to handle the event rate of $400 \mathrm{kHz}$ up to the Storage Handler, so the data flow rate is $2 \mathrm{~TB}$ per second. The amount of recording data is $50 \mathrm{~GB}$ per second.

\subsection{Single-level hardware trigger scheme}

An alternative scheme is also under consideration. As all the detectors are able to accommodate a data readout rate of $1 \mathrm{MHz}$, a single-level hardware trigger scheme is considered and shown in Figure $1-(b)$.

The L0CTP system distributes the Level-0 accept signal at $1 \mathrm{MHz}$ and all data is readout from the detector front-end electronics and transferred to the EF via the DAQ system. The Level-0 trigger system is the same as the two-level scheme described in the previous sub-section. The Level-1 trigger system disappears in this single-level option but the same hardware system as L1Track for regional tracking is moved to the initial step of the EF system to help reduce the rate from $1 \mathrm{MHz}$ to $400 \mathrm{kHz}$, with the data flow into EF increasing to $5 \mathrm{~TB}$ per second.

\section{Level-0 Muon Trigger}

The Level-0 muon trigger (LOMuon) is a part of the ATLAS Level-0 trigger system and a major upgrade on both on-detector and off-detector electronics is planned to profit from the longer Level-0 trigger latency of $10 \mu \mathrm{s}$ and maximum trigger rate of $1 \mathrm{MHz}$. A high-level diagram of LOMuon and DAQ is shown in Figure 2.

The ATLAS muon spectrometer consists of the trigger chambers and the precision trackers. Regarding the trigger chambers, there are Resistive-Plate-Chambers (RPC) in the barrel, Thin-GapChambers (TGC) in the end-cap and the New-Small-Wheel trigger (NSW-trigger) in the end-cap inner station. Regarding the precision trackers, MDTs have been installed in the entire spectrometer as well as NSW-trackers in the end-cap inner station. In addition to the muon chambers, the outermost layer of the sampling calorimeter made of tile-scintillators and iron absorbers (TileCal) has a good signal-to-noise separation for muon signatures and the cell energy is used as input to the muon trigger.

In the endcap of the spectrometer, hit information of TGCs, vector information processed by the NSW-trigger, a 2 out of 3 layers coincidence of the RPCs (BIS-78) and the cell energies of the TileCal are sent to the TGC Sector-Logic board. The TGC Sector-Logic processes trigger signals using a 


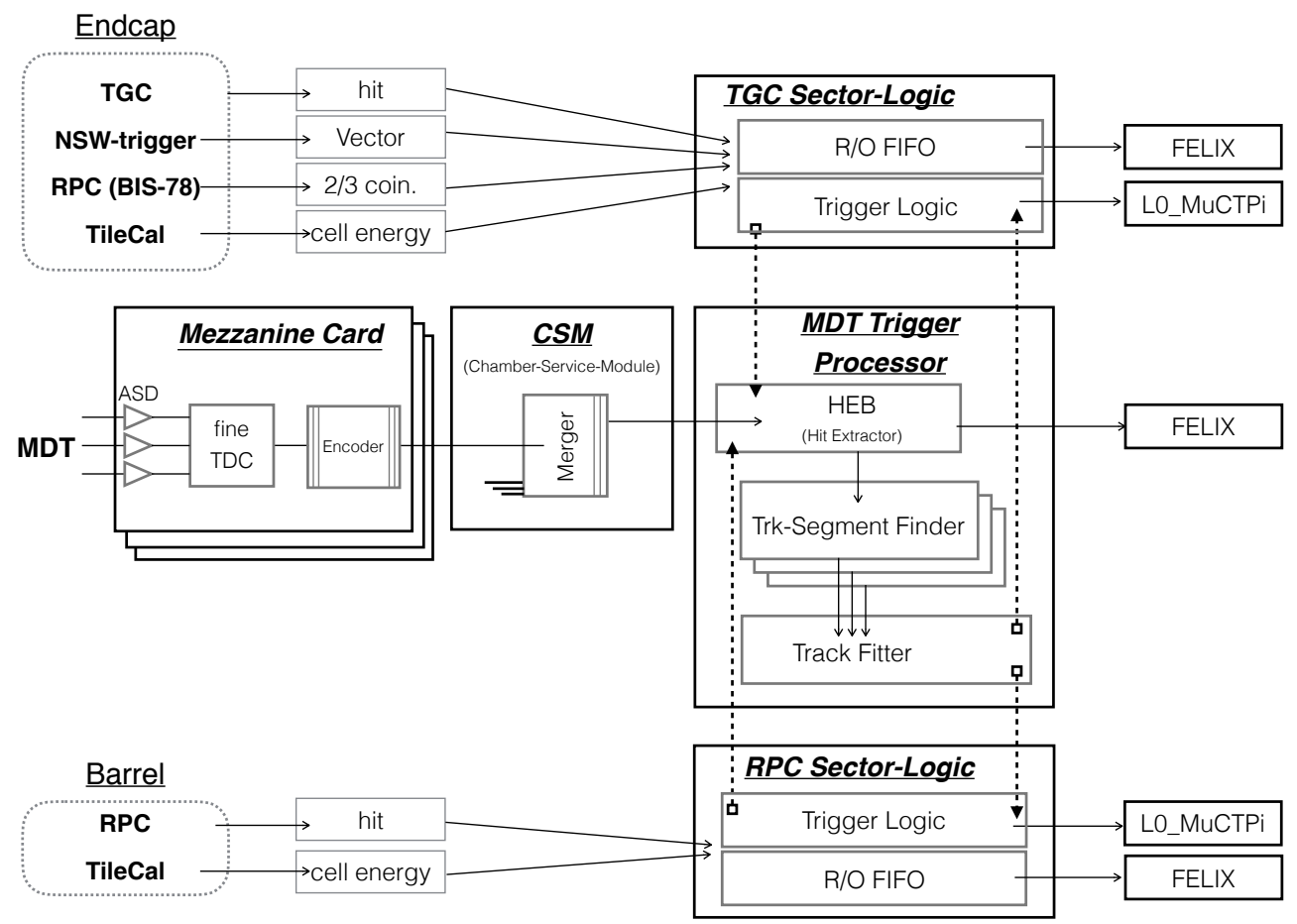

Figure 2. High-level diagram of the Level-0 muon trigger

combination of the coincidence between TGCs and the other detector systems depending on the eta range. The bending angle of the muon track due to the magnetic-field is measured and translated to $p_{T}$, and then compared with thresholds. The estimated $p_{T}$ of the muon track, Region-of-Interest (RoI) and the triggered Bunch-Crossing Identification number (BCID) are passed to the MDT trigger processor.

The barrel trigger system works similarly as the endcap. Hit information of the RPCs as well as cell energies of TileCal are sent to the RPC Sector-Logic board and the $p_{T}$ of the muon track is estimated using the sagitta. The trigger signal is processed and passed to the MDT trigger processor.

To date the MDT has only been used for precision tracking in the ATLAS muon spectrometer because the maximum drift-time of about $800 \mathrm{~ns}$ does not meet the latency allowed for the ATLAS hardware trigger. The use of MDT drift-time information in the Level-0 hardware trigger becomes feasible in the ATLAS trigger and DAQ upgrade because of the longer trigger latency of $10 \mu \mathrm{s}$. The precise spatial information of the MDTs has a significant impact on the momentum resolution, leading to a sharper efficiency turn-on and a consequent reduction of the muon trigger rate.

All the on-detector electronics (Mezzanine cards and Chamber Service Modules (CSM)) will be replaced with new hardware. All the TDC information is sent to the off-detector electronics board, the MDT data processor, via high-speed optical links. In the first step of the MDT data processor, the Hit-Extractor (HEB) selects MDT hits matched to the TGC or RPC RoI. At the same time, TDC infor- 
mation is converted to drift-time according to the BCID triggered by the TGC or RPC. In the second step, track-segments are processed in each MDT station. Several approaches, using Microprocessors on FPGA, introducing Associative-Memory chips and employing a combination of simple coincidence logic and Look-Up-Tables, are proposed and under development. Finally the track-segments from three MDT stations are combined and the transverse momentum of the muon candidate is evaluated. A trigger threshold is applied and the output is sent to the MuCTPi system (LOMuCTPi) via the TGC or RPC Sector-Logic board. According to current studies, the trigger rate is reduced by $70 \%$ with respect to the trigger condition before the HL-LHC program starts, when the trigger threshold is set at $20 \mathrm{GeV}$, as shown in the Figure 3.

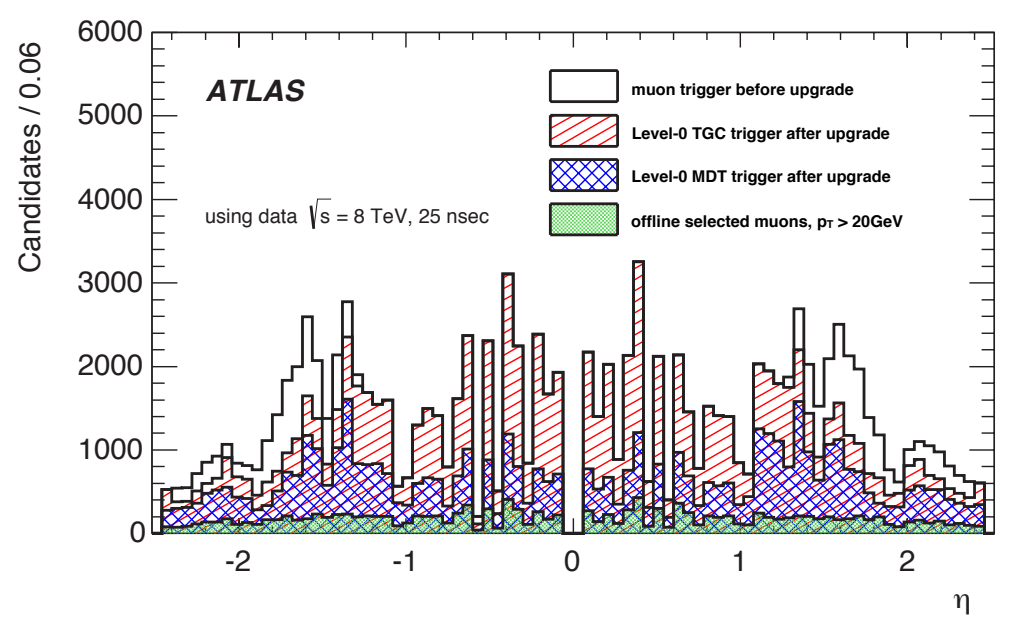

Figure 3. A plot showing $\eta$ distributions of single muon trigger candidates with a $p_{T}$ threshold of $20 \mathrm{GeV} / \mathrm{c}$ [2]. The histogram in white is the situation before the muon trigger is upgraded. Applying a new TGC trigger algorithm in the endcap, the number of entries is reduced by $25 \%$ and the distribution is changed (Red). Further reduction will be expected with the MDT trigger and further trigger rate reduction as shown in the plot (Blue). As a reference, the offline reconstructed tracks having a $p_{T}$ greater than $20 \mathrm{GeV} / \mathrm{c}$ is shown in green.

\section{Level-1 Track-Trigger}

Association of track information with trigger signatures from the calorimeter and muon spectrometers plays a crucial role in improving trigger performance and controlling the trigger rate in the HL-LHC environment. One of the major items in the ATLAS trigger upgrade for the HL-LHC program is a hardware based track trigger aiming to use track information and make a global decision in the ATLAS Level-1 trigger. In order to meet the Level-1 trigger latency of $60 \mu$ s, the track trigger only receives hit information from the pixel and silicon-strip sensors in the inner tracker from the regions around where the Level-0 calorimeter or muon trigger fires (Region-of-Interest : RoI). At the first step of track-finding, multiple pixels or multiple strips are grouped into a single channel, super-cluster (SC). Using an Associative-Memory chip, the hit pattern of SCs of multiple layers is compared with a set of pre-defined track patterns whose transverse momentum is greater than $4 \mathrm{GeV} / \mathrm{c}$. If one of the pre-defined SC pattern matches the input, the hit information with full detector granularity is 
restored and linear fitting is performed to evaluate track information such as transverse momentum, track direction $(\phi, \eta)$ and impact parameter.

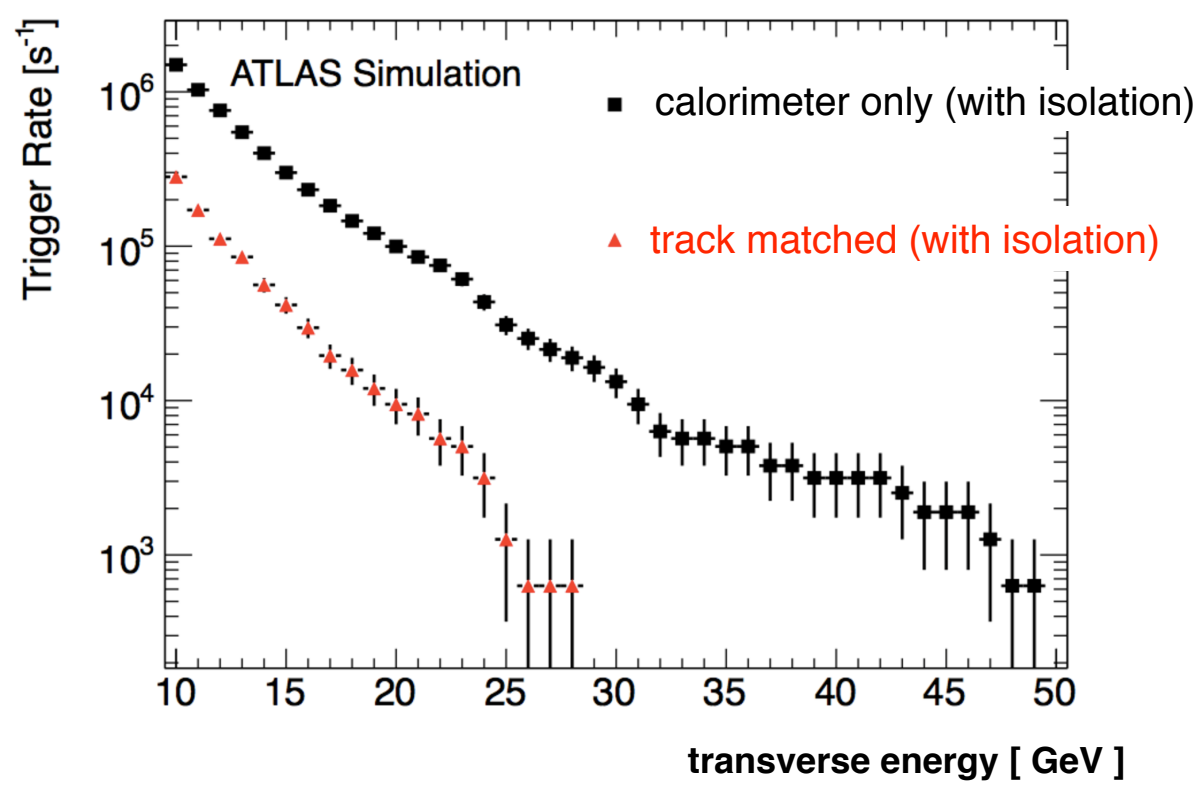

Figure 4. Plot showing expected rates of single electron trigger as a function of the transverse energy observed in the calorimeter [3]. Two different trigger algorithms with and without track information are overlaid in the same plot. A significant trigger rate reduction of a factor of five is found.

Taking the electron trigger as example, the advantage using track information in addition to the calorimeter signature is seen in the first row of Table 1. The ATLAS electro-magnetic calorimeter has fine granularity and electrons are selected based on the lateral shower-shape and a calorimeterbased isolation cut. The expected trigger rate of the Level- 0 isolated single-electron is $200 \mathrm{kHz}$ at the threshold of $22 \mathrm{GeV}$. Requiring a position matching between the calorimeter cluster and the track position as well as the ratio of measured cluster energy over transverse momentum of the track (E/p) to be greater than threshold gives a large suppression of backgrounds from charged hadrons and neutral pions and the Level-1 electron trigger rate is reduced by a factor five to some $40 \mathrm{kHz}$. The single electron trigger rate as a function of $E_{T}$ threshold is shown in Figure 4 for both with and without a requirement of track matching. The study uses simulated minimum bias events with $\mu=70$ and shows a rejection factor of five to ten which may be achieved by requiring a track matching [3]. Further realistic studies under HL-LHC environment, $\mu=200$ need to be performed.

\section{Summary}

The ATLAS trigger and DAQ upgrade for the High-Luminosity LHC program is presented. The target is to keep the physics acceptance in terms of kinematic threshold and geometrical acceptance similar or better than the current trigger conditions. The increased maximum trigger rate as well as 
the longer latency allows to introduce sophisticated algorithms in hardware which have previously been employed in the software based trigger. The Level-0 muon trigger will be totally renewed with information from the precision tracker, MDTs, being introduced in the first step of the hardware trigger. Another new trigger scheme being investigated for the Level-1 trigger is a track trigger using information from the pixel and silicon-strip detectors near the interaction point. The track information is combined with the calorimeter or muon signatures and a trigger decision is taken with an offline-like sophisticated algorithm which leads to a significant trigger rate reduction.

\section{References}

[1] The ATLAS Experiment at the CERN Large Hadron Collider, ATLAS Collaboration, JINST 3 (2008) S08003

[2] ATLAS Phase-II Upgrade Scoping Document, ATLAS Collaboration, CERN-LHCC-2015-020

[3] Letter of Intent for the Phase-II Upgrade of the ATLAS Experiment, ATLAS Collaboration, CERN-LHCC-2012-022 (2013) 